\section{P2-S2.19 ABSTRACT WITHDRAWN}

\section{P2-S2.20 THE ASSOCIATION STUDY OF JOB MOBILITY AND HIV/STI PREVENTION IN FEMALE SEX WORKERS OF HUNAN PROVINCE, CHINA}

doi:10.1136/sextrans-2011-050108.316 associated with low occupational status in the course of jobsseeking and job mobility. Adverse impact were exerted to accessibility of health information and services for FSWs after entering of commercial sex work. The advice of the study is obvious and necessary that except for employment guidance, the students of junior high school should be educated with reproductive health and HIV/STD before graduation in research areas or other rural areas.

Z Zheng, N Jiang. National Center for STD Control, China CDC, Nanjing City, China

Background The job mobility of female sex workers (FSWs) is the working experience that keep low level status and no rising opportunity among all previous jobs before FSWs enter of commercial sex work. To understand the characteristics of job mobility and accessibility to health information and service before engaging in sex work, the study was implemented to its impact on HIV/STD prevention. Strategies and mechanism were explored to provide HIV/STD information and health service to FSWs before they engaging in sex work.

Methods This study was conducted at two cities of Hunan province in September to October, 2009. Ethical approval was obtained from the institutional review board of institute of Dermatology, Chinese Academy of Medical Sciences (CAMS). This study adopted stratified sampling with proportional allocation. Mapping was fulfilled respectively according to distribution of Entertainment Establishment in two cities. FSWs populations were classified into high tier, medium tier and low tier according to the type of working places and the price of sex services. Participants were determined after setting up sampling framework. Data were collected through questionnaire survey and in-depth interview and questionnaire survey on the basis of informed consent written by participants. The data were double entered, using Epidata software, by two researchers independently and were checked to assure accuracy and completeness. Statistical analysis of data was performed with SAS version 9.1 (SAS Institute Inc, Cary, NC). Univariate and multivariate analysis were used to examine for associations between variables of interest.

Results 207 FSWs were recruited in Zhangjiajie city and Shimen county of Hunan province. Of all respondents, the median age was 22 (range 16-48), 72.5\% were unmarried, 67.8\% had junior high school education, and $75.6 \%$ were from rural areas. $65.3 \%$ of the respondents once migrated to one or more places outside original hometown for job-seeking. $15.2 \%$ of 207 respondents reported sex work as their first job, while the rest reported at least one job before engaging in sex work. No significant differences were found among monthly salary of all previous jobs before sexual services. Significant differences were found between average monthly salary of previous jobs and sexual services at two sites. The median monthly income of sex work was about four times as much as that of their all previous jobs. $83.7 \%$ of the respondents were engaged in individual business or private enterprise before sexual services, especial for working in service industry or factory. In the opinion of job-seeking way and approach, $14.7 \%$ of the respondents had obtained employment information from regular channel or official agent of labour management department etc. Only $28.1 \%$ of the respondents had gained HIV/STD prevention information before sexual services. $89.7 \%$ of the respondents were not provided any counselling or reproductive health services in the course of job mobility, however $67.5 \%$ of the respondents would be eager for receiving periodic and accessibility of reproductive health services.

Conclusions Job mobility of FSWs before sex work is objective phenomenon at research areas. Occupational status and income is low level and poor work conditions of all previous jobs before sex work. It existed impossible rising opportunity between different jobs and positions. That is the horizontal characteristics of job mobility for FSWs population. It is significant association between low occupational status and lack of health relevant information and service, while poor accessibility to health information and services is

\section{P2-S2.21 TRADITIONAL DEVADASI SYSTEM UNDER TRANSITION: BOON OR BANE FOR HIV PREVENTION PROGRAMIIE?}

doi:10.1136/sextrans-2011-050108.317

${ }^{1} \mathrm{~K}$ Gurav, ${ }^{2} \mathrm{E}$ Cooper, ${ }^{2} \mathrm{~J}$ Junno, ${ }^{2} \mathrm{D}$ Stein, ${ }^{1} \mathrm{D}$ R Chintada, ${ }^{1} \mathrm{M}$ Doddamane, ${ }^{2} \mathrm{~S}$ Moses. ${ }^{1}$ Karnataka Health Promotion Trust, Bangalore, India; ${ }^{2}$ University of Manitoba, Winnipeg, Canada

Background One of the key factors attributed to high prevalence of HIV in Northern Karnataka (in India) is socially accepted and widely practiced sex work. Girls from "Devadasi" community enter into sex work through socially accepted way of being dedicated to deity "Yellamma". Currently, the "Devadasi" system is under transition where, new dedications are not accepted by community leaders. As a result, the social organization of traditional sex work is changing. Methods An exploratory research was undertaken in the district of Bagalkot, Karnataka. Ethnographic methods, including participant observation and Focus Group Discussions (FGDs) were used. FGDs were conducted with leaders, sex workers, peer educators, adolescent girls from "Devadasi" community and HIV service providers. Results "Daiva" is a committee consisting of key leaders from "Devadasi" community. "Daiva" functions at the local (village) level and make decisions for the welfare of their community. The death of many young "Devadasi" sex workers due to AIDS was reported as a key factor for "Daiva" in stopping girls from entering sex work. Towards this end, "Daiva" has declared that the families who dedicate their daughters will be fined (RS 5000 to 20000) and be outcasted. Although there seem to be a consensus to the decision of "Daiva", there are families who still want their daughters to start sex work. The fear of social and financial reprimand is placing new sex workers and their families in a marginalised and potentially illegal position within their village. Meanwhile, it is making new "Devadasi"? sex workers, conceal their identity and also avoid the HIV prevention services offered by peer educators of their own community at the door step.

Conclusion Changing social organization and conditions of "Devadasi" sex workers increases their intrinsic risk and vulnerability to HIV. Therefore it is important to address the structural changes occurring within the "Devadasi" community and also create an environment for new sex workers to freely access HIV prevention and care services. An innovative intervention model that could negotiate between objectives of "Daiva" and needs of sex workers is required."

\section{P2-S2.22 INNOVATIONS TO ATTRACT YOUNG FEMALE SEX WORKERS TO ACCESS STI SERVICES IN DROP IN CENTRES (DIC): A CASE STUDY FROM BANGALORE, SOUTH INDIA}

doi:10.1136/sextrans-2011-050108.318

${ }^{1} \mathrm{E}$ Michael, ${ }^{2} \mathrm{~S}$ K Murugan, ${ }^{1} \mathrm{~L}$ Viswanatha, ${ }^{3} \mathrm{R}$ Pushpalatha. ${ }^{1}$ Karnataka Health Promotion Trust, Bangalore, India; ${ }^{2}$ University of Manitoba, Winnipeg, Canada; ${ }^{3}$ Swati Mahila Sangha, India

Background Young female sex workers pose a challenge in accessing continuous services in prevention program. Being young, relatively active and new to sex work increases their vulnerability drastically but also attract more clients. As young sex workers they often do not access services provided in the drop in centre due to several 